\title{
Comparison of Gabor Filter Bank and Fuzzified Gabor Filter for License Plate Detection
}

\author{
Vladimir Tadic, Zoltan Kiraly, Peter Odry
}

University of Dunaújváros, Táncsics Mihály u. 1/A, 2401 Dunaújváros, Hungary, tadityv@uniduna.hu,kiru@uniduna.hu,podry@uniduna.hu

\section{Zeljen Trpovski, Tatjana Loncar-Turukalo}

University of Novi Sad, TrgDositejaObradovića 6, 21000 Novi Sad, Serbia, zeljen@uns.ac.rs, turukalo@uns.ac.rs

Abstract: This paper presents a comparative study of improvements to the algorithms for license plate extraction from images captured using conventional, modest-quality cameras. It compares the results and efficacy of two similar algorithms which primarily differ in the raw image pre-processing stage of the initial image. One algorithm uses the Gabor filter bank with distinctly crisp parameters; the other relies on the fuzzified Gabor filter bank with fuzzified parameters to facilitate their adjustment. Results indicate that the fuzzy reasoning introduced for Gabor filter parameter adjustment improves the detection of the components of interest in complex images and adds minimal deviation compared to the Gabor filter bank with distinctly crisp parameters.

Keywords: Gabor Filter; Filter Bank; Crisp Parameters; Parameter Fuzzification; License Plate Detection

\section{Introduction}

\subsection{Background}

One of the most common uses of object recognition/computer vision is an automatic detection and recognition of license plates. Many systems, such as those used in industry, parking services, airports, customs control, the military, and the police, contain on board software for license plate recognition. An additional application of license plate recognition is in control systems in areas with limited accessibility (embassies, factories, police and military facilities) and for identifying lost or stolen cars [1]. 
The recognition of license plate's characters falls under the more general and well explored problem of characters' segmentation and recognition. There are numerous algorithms with an excellent performance that can be easily adapted to any license plate recognition system [2]. However, the extraction of a license plate from an image still remains a highly researched issue within object recognition, with many papers still being published on the topic [1], [3], [4], [5].

The goal of this research is to compare and evaluate two robust algorithms that achieve good performance on the images taken with commercial cameras with all the problems inherent to this image acquisition method. In commercial applications, such as low enforcement, the images are acquired with special REG (special register) cameras that combine special and expensive optics with preprocessing software. The images captured with commercial digital cameras have numerous flaws such as reflections, shadows, and noise, resulting in a poor image quality. The compared algorithms would enable the use of affordable cameras and less strictly controlled scene for a license plate detection.

In this paper the performance and efficacy of two similar algorithms, with the main difference in the pre-processing step, are compared and contrasted. Wellconceived pre-processing step can compensate for flaws in an image acquisition, or ambient conditions, facilitating easier extraction of a license plate from an image in the later stages. The first algorithm used a Gabor filter bank with distinctly crisp parameters (denoted GFBank) [5], while the second algorithm relied on a fuzzified Gabor filter bank (denoted FuzzyGF) [1] with the parameters fuzzified to maximally adjust the filter's response. The problem of filtration using Gabor filters has been accessed by taking into account fuzzy logic, a powerful mathematical apparatus [6], [7], [8] that closely imitates human reasoning. Using this apparatus, the Gabor filter parameters were converted into fuzzy parameters to improve the filter's response [9], [10]. The results of this filtering contribute to more robust detection and extraction of license plates from complex vehicle images.

There are several relevant variables influencing the performance of a license plate detection systems: illumination, quality of the image acquisition system, capturing conditions (distance, angle), and variability in license plate dimensions. However, the main challenge with a plate extraction is the skewness and the presence of extra components of various dimensions similar to a license plate. In such cases, invalid detection can occur, and the wrong component may be extracted. Poor image capture conditions include a greater angle deviation from the regular $90^{\circ}$, and insufficient or excessive capture distance. Sufficient or excessive illumination is also of significant importance for a successful performance. The algorithms compared in this work are resistant to poor capture conditions if the relevant parameters are set within the acceptable limits. It is important to emphasize that the use of Gabor filters provides excellent results in both cases, as Gabor filters successfully handle noise, poor illumination, and different artefacts in the image. These features are of uttermost importance since our initial motivation was 
improving the license plate extraction performance for a low-cost image acquisition regardless of illumination conditions.

The rest of this article is organized as follows. The literature overview on this topic is covered in Section 1.2. The Gabor filter and the fuzzy methods are briefly introduced in Section 2. The comparison of license plate detection algorithms, the GFBank and the FuzzyGF is described in Section 3. The validation of the algorithms is explained in Section 4 followed by the concluding remarks.

\subsection{Recent Works}

Many different procedures for number plate detection and extraction have been recently proposed, such as the fuzzified Gabor filter [1], the method based on the wavelet transform [11], attribute filtering [12], edge detection and noise reduction [13], and mathematical morphology [14]. Each of these approaches has its own limitations and advantages [15], [16], [17]. The quality of the cropped license plates resulting from these procedures is primarily influenced by the quality of the camera that captures the source image and by the quality of the algorithm used for the image processing.

Corneto et al. [18] used a Haar classifier for a plate detection and extraction followed by plate segmentation. The results indicate that the computational cost and accuracy rate considering this approach were acceptable to real time applications, with an execution time less than one second. Babu $\mathrm{K}$ et al. [19] applied a Sobel mask for plate region localization followed by morphological filtering and connected components analysis. The plate region was extracted using a bounding box and a template matching method relying on a correlation for recognition of each character in the number plate. Do et al. [2] developed an Android program that processes the image acquired by the built-in camera of a mobile device to capture the license plate number and save it into a database for subsequent applications. They imported open-source Open CV libraries into the project for selected image processing steps to reduce the programming time. The Tesser act engine and neural network were used for optical character recognition (OCR) to convert the license plate image into machine-encoded text. Qiu et al. [20] integrated color and edge detection methods to increase the success rate of locating license plates. They employed connected component analysis and vertical projection methods alternatively to improve the precision and efficiency of segmentation. For character recognition, they applied an improved K-Nearest Neighbours algorithm. Their experimental results indicate that the optimized system had a high license plate recognition rate with an accuracy of $96.75 \%$. Roy et al. [21] developed an algorithm for detection and recognition of Bangladeshi license plates. They noted that the variations among the license plate patterns and complex background of Bangladeshi license plates made it difficult to use the existing algorithms. They selected the portions of green color for matching RGB intensity of the plate. A boundary-based contour algorithm, area, and aspect ratio 
were used to locate the license plate in the vehicle region. License plate rows containing registration information were separated using horizontal projection with the threshold value. The characters and digits of the rows were segmented using vertical projection with the threshold value. Finally, template matching was used for recognizing [22], [23], [24] the characters and digits of the license plate. Wang et al. [25] used the Roberts operator for edge detection and morphological erosion, image clustering and region filling for plate detection from complex backgrounds. They used vertical projection for plate segmentation and a back propagation neural network for character recognition. They reported a $97.80 \%$ correct license plate recognition rate. Yuan et al. [26] presented a robust and efficient method for license plate detection with the purpose of accurately localizing vehicle license plates from complex scenes in real time. A simple effective image downscaling method was first proposed to substantially accelerate license plate localization without sacrificing detection performance compared with that achieved using the original image. Then, a novel line-density filter approach was proposed to extract the candidate regions, thereby significantly reducing the area to be analyzed for license plate localization. Moreover, a cascaded license plate classifier based on linear SVMs using color saliency features was introduced to identify the true license plate from among the candidate regions. Extensive experiments on the widely used Caltech license plate dataset and their introduced dataset demonstrate that the proposed approach substantially outperformed stateof-the-art methods in terms of both detection accuracy and run-time efficiency, increasing the detection ratio from $91.09 \%$ to $96.62 \%$ while decreasing the run time from $672 \mathrm{~ms}$ to $42 \mathrm{~ms}$ for processing an image with a resolution of $1082 \times$ 728. Panchal et al. [27] considered feature extraction using the Harris corner detection algorithm. An aspect ratio limit was set to restrict the license plate viably subsequent to separating each single corner point and using a scanning window approach for plate extraction. Morphological operations were performed in the plate segmentation stage. The overall accuracy of the proposed method was 93.84\%. Xie et al. [28] presented a novel convolutional neural network (CNN) based method for high-accuracy real-time car license plate detection. They proposed a CNN-based MD-YOLO (Multi-Directional "You Only Look Once") framework for multi-directional car license plate detection. They also introduced the prepositive CNN model, ALMD-YOLO (attention-like MD-YOLO) method which performs better than the single MD-YOLO. These solutions can elegantly solve the problem of multi-directional car license plate detection, and can also be deployed easily in real-time circumstances, because of their reduced computational complexity.

Table 1 summarises the above mentioned approaches, main methodology used, database size and the rate of successful localizations. The direct comparison is hampered by the different databases used, and various assumptions and limitation introduced in the methodological steps. The universally applicable algorithm, irrespective of image quality, illumination, capturing settings and licence plate type is still a challenging task. 
Table 1

Summary of the mentioned approaches

\begin{tabular}{|l|l|c|c|}
\hline \multicolumn{1}{|c|}{ Related Work } & \multicolumn{1}{|c|}{ Algorithm } & Database size & $\begin{array}{c}\text { Correctly } \\
\text { localized }\end{array}$ \\
\hline \hline Tadic et al. [1] & FuzzyGF & 718 & $97.9 \%$ \\
\hline Yu et al. [11] & Wavelets & 765 & $97.91 \%$ \\
\hline Yimyam et al. [13] & $\begin{array}{l}\text { Edge detection and noise } \\
\text { reduction }\end{array}$ & 50 & $82 \%$ \\
\hline Jabar et al. [14] & Mathematical morphology & 100 & $84.19 \%$ \\
\hline Corneto et al. [18] & Haar classifier & 1410 & $92.55 \%$ \\
\hline Babu K et al. [19] & Sobel mask & 45 & $93.33 \%$ \\
\hline Do et al. [2] & Neural network & 200 & $96 \%$ \\
\hline Qiu et al. [20] & $\begin{array}{l}\text { Integrated color and edge } \\
\text { detection methods }\end{array}$ & 400 & $96.75 \%$ \\
\hline Roy et al. [21] & $\begin{array}{l}\text { Boundary-based contour } \\
\text { algorithm }\end{array}$ & 180 & $93 \%$ \\
\hline Yuan et al. [26] & Line-density filter & 126 & $96.62 \%$ \\
\hline Panchal et al. [27] & Harris corner detection & 65 & $93.84 \%$. \\
\hline Xie et al. [28] & Convolutional neural network & 2049 & $99.5 \%$ \\
\hline
\end{tabular}

In the majority of studies in this field, the initial license plate images were captured with special register (REG) cameras designed for license plate recognition or with high-quality cameras. These cameras are considerably more expensive than commercial automatic digital cameras. REG cameras combine special optics with software support that contributes significantly to the development of high-quality initial images [1], [5].

The fuzzy set theory was a cornerstone in numerous papers dealing with numerous application scenarios. In the following is a brief overview of the papers related to fuzzy logic. Várkonyi-Kóczy [29] and Moya-Albor et al. [30] successfully applied fuzzy logic to corner and edge detection. Cirneanu et al. [31] proposed a supervised technique for the detection and localization of the optic disc in retinal images. The proposed processing technique is based on Discrete Fourier Transform and Gabor filters. Devasenapati [32] et al. in their paper evaluates the use of fuzzy unordered rule induction algorithm (FURIA) with correlation-based feature selection embedded feature subset selection as a tool for misfire detection. From the results obtained the authors conclude that the combination of statistical features and FURIA algorithm is suitable for detection of misfire in spark ignition engines. Haidegger et al. [33] described the minimalistic models that were tested with linear and PID-fuzzy control options to provide a simple, universal and scalable solution for the challenges of tele-surgery over large distances. Jocic et al. [34] proposed an algorithm for discovering similar nodes in very large directed graphs, with millions of nodes with billions of connections, which is based on the 
fuzzy set theory. Vrkalovic et al. [35] presented a model-free sliding mode controllers and Takagi-Sugeno fuzzy controllers for the flux and conductivity control of Reverse Osmosis Desalination Plants.

\section{Methods}

\subsection{Gabor Filter}

Gabor filters are extensively used for texture detection and extraction because of their optimal localization properties in both spatial and frequency domain. When a Gabor filter is applied to an image, it gives the highest response at points and at edges where texture changes. By virtue of these characteristics, algorithms based on Gabor filters are successfully applied in computer vision applications [31] such as license plate detection, face detection, texture extraction, character recognition and others [1], [5].

The complex Gabor function in a spatial domain is represented as follows:

$g(x, y)=s(x, y) w_{r}(x, y)$

where the function $s(x, y)$ represents the complex sine wave and the function $w_{r}(x, y)$ represents the 2D Gaussian.

The complex sine wave is defined as:

$s(x, y)=e^{j\left(2 \pi\left(u_{0} x+v_{0} y\right)+\varphi\right)}$

where the $\left(u_{0}, v_{0}\right)$ denotes spatial frequencies and the $\varphi$ is the phase of the filter.

This complex sine wave can be split into the real and imaginary part as follows:

$\operatorname{Re}(s(x, y))=\cos \left(2 \pi\left(u_{0} x+v_{0} y\right)+\varphi\right)$

$\operatorname{Im}(s(x, y))=\sin \left(2 \pi\left(u_{0} x+v_{0} y\right)+\varphi\right)$

Parameters $\left(u_{0}, v_{0}\right)$ represent spatial frequencies in Cartesian coordinates. These spatial frequencies can be represented in polar coordinates as follows:

$f=\sqrt{u_{0}^{2}+v_{0}^{2}}$

$\Phi=\operatorname{arctg}^{-1}\left(\frac{v_{0}}{u_{0}}\right)$

and the spatial coordinates are expressed as:

$u_{0}=f \cos \Phi$

$v_{0}=f \sin \Phi$ 
Using the previous equations, the complex sine wave is represented as:

$s(x, y)=e^{j(2 \pi f(x \cos \Phi+y \sin \Phi)+\varphi)}$

If the 2D Gaussian is defined as:

$w_{r}(x, y)=A e^{-\left(\frac{\left(x-x_{0}\right)^{2}}{2 \sigma_{x}^{2}}+\frac{\left(y-y_{0}\right)^{2}}{2 \sigma_{y}^{2}}\right)}$

where $A$ is the amplitude, $\left(x_{0}, y_{0}\right)$ are the centre of the function and $\sigma_{x}$ and $\sigma_{y}$ denotes the deviation of the Gaussian by each of the spatial coordinates.

After preliminary consideration, a function $g(x, y, f, \Phi, \sigma)$ which represents the Gabor filter with the orientation of $0^{\circ}$ where the $f$ is the spatial frequency and the $\Phi$ denotes the filter orientation is obtained as follows [1], [5]:

$$
g(x, y, f, \Phi, \sigma)=A e^{-\left(\frac{\left(x-x_{0}\right)^{2}}{2 \sigma_{x}^{2}}+\frac{\left(y-y_{0}\right)^{2}}{2 \sigma_{y}^{2}}\right)} e^{j(2 \pi f(x \cos \Phi+y \sin \Phi)+\varphi)}
$$

As it can be seen, the expression (11) is a developed form of the expression (1) [1], [5], [10].

The first exponential function represents the 2D Gaussian; the second exponential function represents the $2 \mathrm{D}$ complex sinusoid. Again, the $A$ denotes the amplitude, $\left(x_{0}, y_{0}\right)$ is the center of the function, $\sigma_{x}$ and $\sigma_{y}$ are the standard deviations, $f$ is the spatial frequency, $\varphi$ is the phase and $\Phi$ is the orientation of the Gabor filter.

It has been shown, that the deviation of the filter can be represented via the spatial frequency $f$ as follows [1], [5]:

$\sigma_{x}=\lambda k_{x}$ and $\sigma_{y}=\lambda k_{y}$

In the previous expressions $k_{x}$ and $k_{y}$ represent scaling factors for tuning the bandwidth of the filter. The wave length $\lambda$ is in pixels and it is related with the spatial frequency as [1], [5]:

$f=\frac{1}{\lambda}$

All these parameters are essential in forming the Gabor filter for appropriate filtering [1], [5].

\subsection{Gabor Filter Bank with Crisp Parameters}

With further development, Gabor filter banks are created, which usually consist of Gabor filters with various scales, frequency and orientations. This concept makes it possible to use a larger number of values for each filter parameter in order to improve the quality of the Gabor filter's response [5]. 
In the initial research, the first developed Gabor filter bank has variable crisp parameters, wavelength and angle [5]. Both the wavelength and the angle have 17 values each, stored in two separate arrays. These parameters are obtained empirically [5]. Finally, the filter bank's final response is obtained by summing the results of all individual filter responses within the filter bank. The effect and the response of this filter bank is presented in the next sub-section on examples of license plate extraction.

Table 2

Gabor filter bank parameters

\begin{tabular}{|c|c|}
\hline Parameters & Values \\
\hline wavelength & 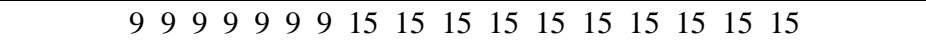 \\
\hline angle & $\begin{array}{ccccccccccccccccc}-3 & -2 & -1 & 0 & 1 & 2 & 3 & -90 & 90 & 91 & -91 & 92 & -92 & 89 & -89 & 88 & -88\end{array}$ \\
\hline $\mathrm{k}_{\mathrm{x}}$ & $\begin{array}{lllllllllllllllll}0.5 & 0.5 & 0.5 & 0.5 & 0.5 & 0.5 & 0.5 & 0.5 & 0.5 & 0.7 & 0.7 & 0.7 & 0.7 & 0.7 & 0.7 & 0.7 & 0.7\end{array}$ \\
\hline $\mathrm{k}_{\mathrm{y}}$ & $\begin{array}{lllllllllllllllll}0.5 & 0.5 & 0.5 & 0.5 & 0.5 & 0.5 & 0.5 & 0.5 & 0.5 & 0.7 & 0.7 & 0.7 & 0.7 & 0.7 & 0.7 & 0.7 & 0.7\end{array}$ \\
\hline
\end{tabular}

\subsection{Fuzzified Gabor Filter}

Another approach in the development of license plate detection algorithms was the inclusion of fuzzy reasoning [1]. The reason for this is the ability of fuzzy logic to express the degree of uncertainty in human thinking. Namely, it was noticed during the experiments [5] that the algorithm with the Gabor filter bank with crisp parameters has detection difficulties when the license plate is at a very skewed angle, is slanting or when the size of the plate excessively varies in the input image. The wavelength and the orientation of the Gabor filter are fuzzified. The values of the intervals for input and output membership functions were empirically obtained [1].

In the fuzzification process a Bell type membership function has been selected as the input membership function in interval [-10 10]. This membership function smoothly and equally describes the angles of interest.Namely, the angle of $0^{\circ}$ is the central angle around which it is necessary to detect vertical components, but due to the imperfection of the input image, some adjacent angles come into consideration too, whose influence decreases from zero around both sides according to the Bell's function. This can be expressed with the formula [9], [10]:

$$
\mu(z)= \begin{cases}S\left(z, \phi_{1}, \phi_{2}\right) & z<\phi_{2} \\ S\left(2 \phi_{2}-z, \phi_{1}, \phi_{2}\right) & \phi_{2} \leq z\end{cases}
$$

where the interval of the angles is $\phi \in\left[\phi_{1}, \phi_{2}\right]$. In this research, the end values of the interval were empirically chosen as -10 and 10 , and the function $S$ is given by the equation (14) [9], [10]. 
To improve the filtering, the interval of all possible orientations of the filter should be small, or a smaller number of adjacent angles should be used to facilitate the localization of the license plate in the later stages. For these purposes, the triangular shape membership function is used. In this case the interval of the output membership function is [-3 3], which corresponds to the need for small angle deviations from the $0^{\circ}$ and it is obtained that $\mu\left(\phi_{\text {crisp }}\right)=1$, where the $\phi_{\text {crisp }}$ is the maximum value of the triangular membership function for the angle of $0^{\circ}$. The start and end points of the interval are chosen as [1], [9], [10]:

$$
\phi_{\text {crisp }}=\frac{\phi_{i}+\phi_{f}}{2}
$$

and the corresponding interval is selected as $\phi_{f}-\phi_{i}=6^{\circ}$. The Fig. 1 (a) and Fig. 1 (b) shows both membership functions. It is noticeable that narrowing exists around the zero in Fig. 1 (b).

In this way, the appropriate input and output membership functions have been defined. In order to obtain the fuzzy system and complete the fuzzification process, the IF-THEN rules have to be defined. The complete procedure for fuzzification was simplified using the built in functions based on literature [9], [10]. The IF-THEN rules were generated automatically using the functions provided by Gonzales et al. [9], [10] (function fuzzysysfcn) without any additional interfaces. After the defuzzification, an approximation was applied using the builtin lookup table from the literature [9], [10]. The resulting fuzzy system for the angles is presented in Fig. 1 (c).

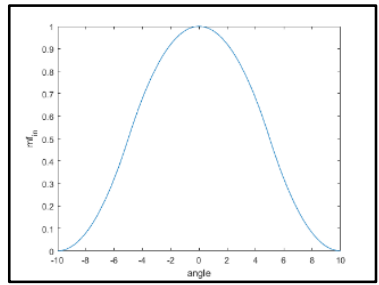

(a)

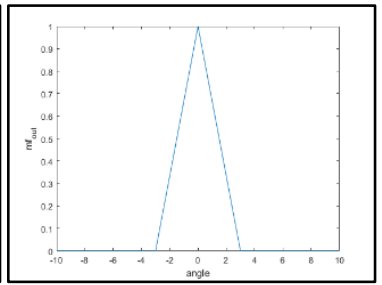

(b)

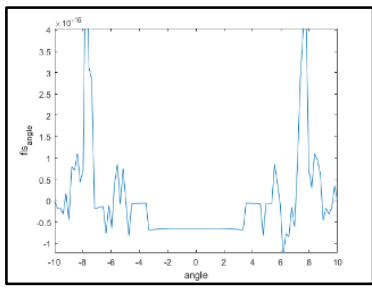

(c)

Figure 1

(a) Input membership function for the angles; (b) output membership function for the angles; (c) fuzzy system for the angles

For the fuzzification of the wavelengths the procedure was almost identical with some minor changes, which arise primarily from the nature of the problem.

It has been experimentally obtained that the spatial wavelength's range is from 9 and 15 pixels and the fuzzification has been carried out around these values. For input membership function a generalized Bell's function was used, with two inputs and two intervals that cover the input domain. The intervals centered around 9 and 15 are $[7,11]$ and $[13,17]$, respectively (Fig. 2(a)). 
Two output segments and the triangular membership function were used to describe the output and achieve the smallest deviations from the central wavelength values. For the central input values of 9 and 15 pixels, the selected intervals were $[8,10]$ and $[14,16]$. These functions have fully described the input and the output of the fuzzy system.

After defining the IF-THEN rules and the fuzzification of the system, a new fuzzy system was obtained that describes the wavelength changes. Fig. 2 (a) and Fig. 2 (b) present the input and output membership functions defined above certain intervals of interest. Finally, the Fig. 2 (c) shows the fuzzy system for the wavelengths.

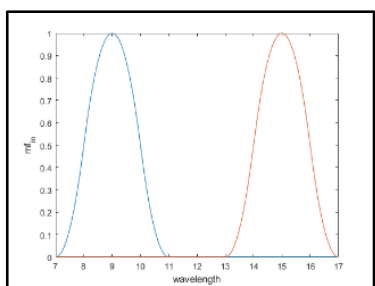

(a)

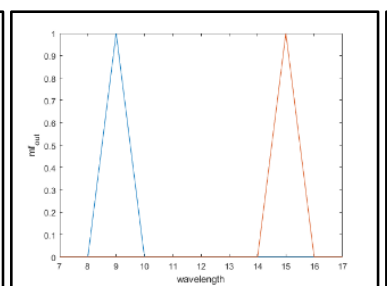

(b)

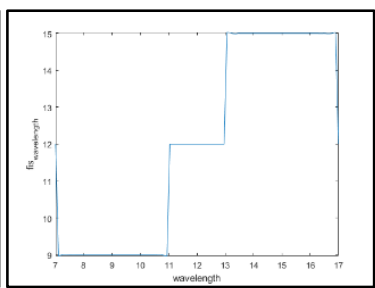

(c)

Figure 2

(a) Input membership function for the wavelengths; (b) output membership function for the wavelengths; (c) fuzzy system for the wavelengths

Once the fuzzification of the Gabor filter had been completed, the new Gabor filter bank has been developed with fuzzified parameters. Having filtered the input images with the fuzzified Gabor filter, the detection rate has been increased [1]. Also, the effect and the response of the fuzzified Gabor filter bank is displayed at a later stage in the next chapter on an exemplary image.

\section{Comparison of the License Plate Detection Algorithms}

In the following section, the performance of two algorithms for license plate detection will be compared. The processing pipeline introducing the order and roughly indicating the effect of each algorithm step in an exemplary image is presented in Fig. 3. The only significant difference between the two algorithms is in the pre-processing block performing initial filtering with the specific Gabor filter [1], [5]: Gabor filter bank with the constant parameters (GFBank) and fuzzy Gabor filter (FuzzyGF). After the pre-processing step, both algorithms follow the same pipeline: a sequence of morphological operations, followed by edge detection, threshold application, detection of the frame, plate marking, and cropping. In the following the common algorithms steps will be presented (Fig. 3). 


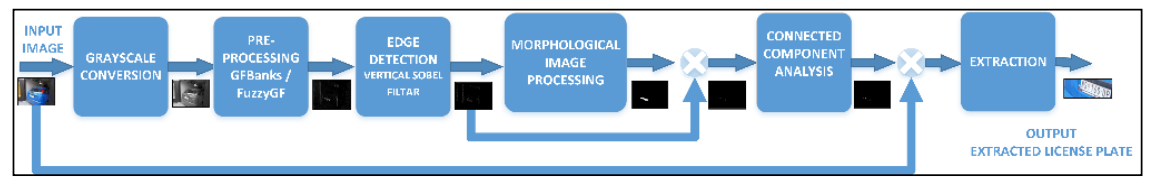

Figure 3

The processing pipeline of the algorithms

After the input image was read and rescaled, the image was grayscaled. This is followed by filtering using the GFBank or the FuzzyGF. The results and a comparison of the two algorithms, i.e., the influence of both versions of the Gabor filters on the initial image, are described in the example below.

The image of the vehicle in Fig. 4 (a) was captured at a rather sharp angle, at daylight, in sunny weather, without an artificial light source. The image was taken with a 2-Megapixel camera on an old mobile phone (Nokia C2). It is clearly visible that the image is slightly blurred due to the sunrays and weather conditions. Fig. 4 (b) displays the same image, in grayscale.

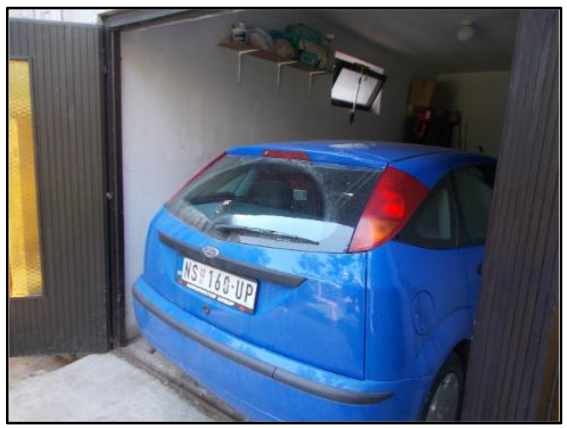

(a)

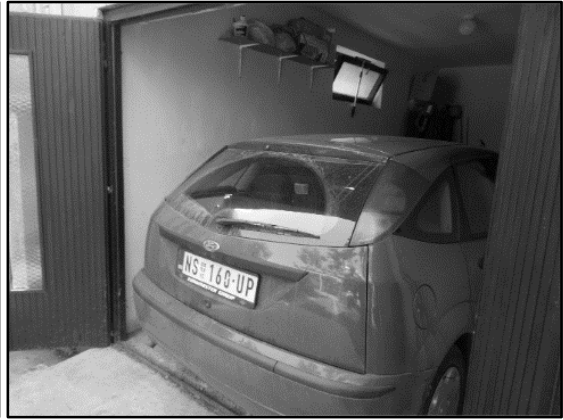

(b)

Figure 4

(a) Original colored image and (b) grayscaled image

Fig. 5 (a) displays the response of the GFBank with clearly distinct parameters [5] and (b) the response of the FuzzyGF [1]. As it can be observed, the response is the strongest in the area around the license plate itself; however, even in this region, some darker textures remain.

The response of the image to the fuzzified Gabor filter (Fig. 5 (b)) is the strongest in the area around the plate, however, with fewer dark textures immediately around the plate, which is an improvement achieved with the FuzzyGF. 


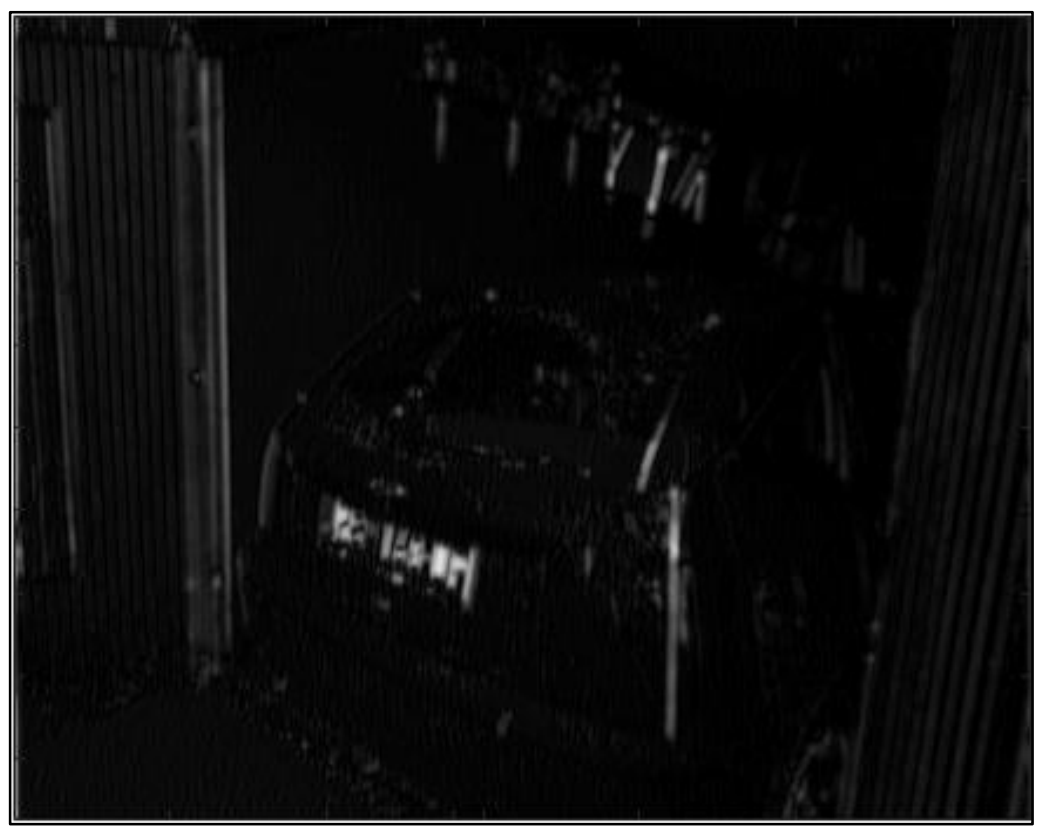

(a)

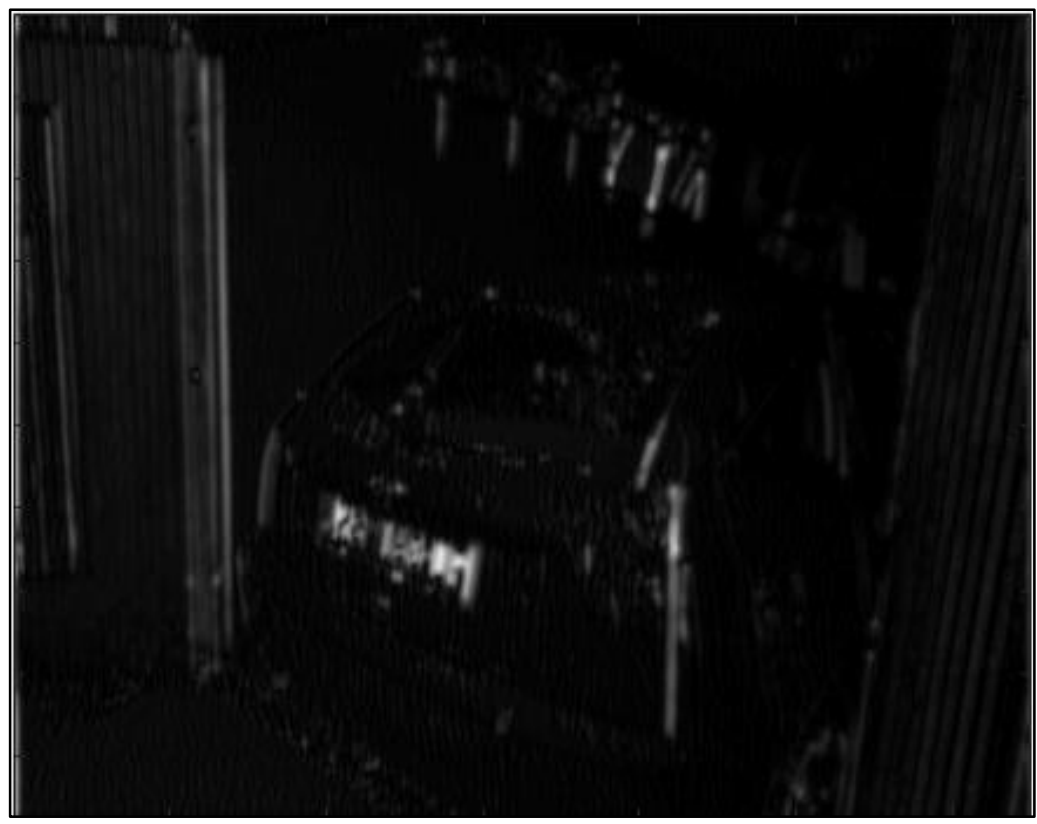

(b)

Figure 5

(a) GFBank response and (b) FuzzyGF response 
After filtering, the next step was edge detection using a vertical Sobel operator [1], [5], [9]. Fig. 6 presents the images after the edge detection for two compared algorithms with different pre-processing step:(a) in case of the GFBank preprocessing, (b) case of the FuzzyGF pre-processing. In both results, there are tiny, dense components of interest in the area around the license plate. This is a promising result towards a more successful final plate detection.

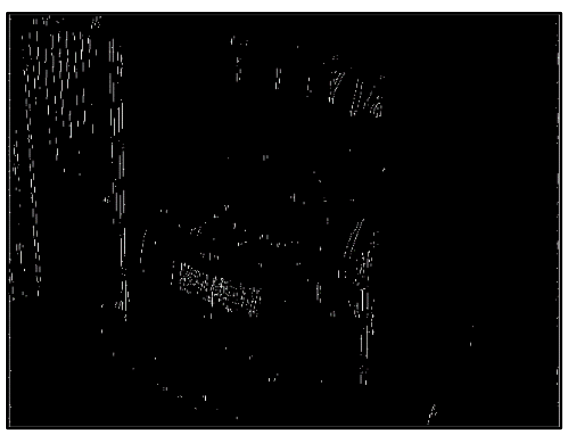

(a)

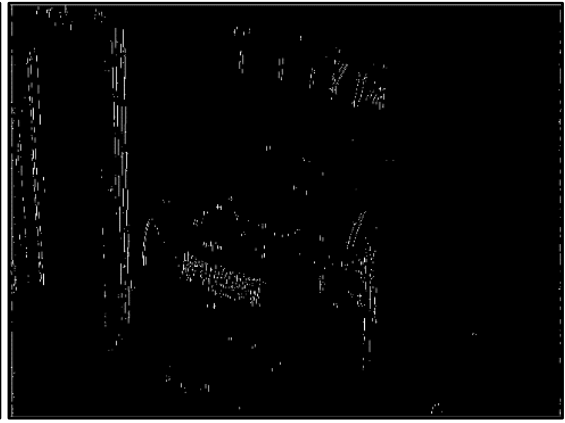

(b)

Figure 6

Result of edge detection to: (a) GFBank response and (b) FuzzyGF response

After edge detection, a sequence of several morphological operations was performed and an algorithm for the analysis of connected components was applied [1], [5], [9], [19]. The objective of this step is to remove the unnecessary parts of the image and to form a binary mask of the license plate region, which facilitates the extraction of the plate from the complex vehicle image and its surroundings [1], [5], [10].

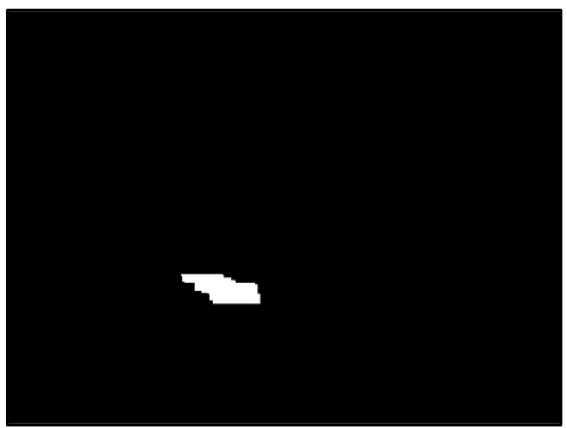

(a)

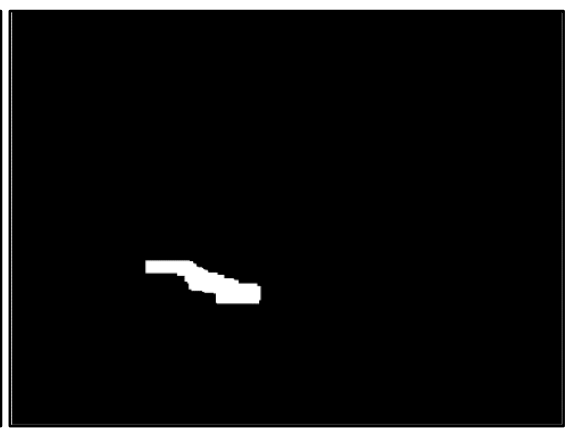

(b)

Figure 7

(a) Binary mask of the algorithm with the GFBank and (b) binary mask of the algorithm with the FuzzyGF

Fig. 7 displays the resulting binary masks of both approaches. It is clearly visible that the mask is somewhat narrower in the left image (GFBank) than on the right (FuzzyGF). 


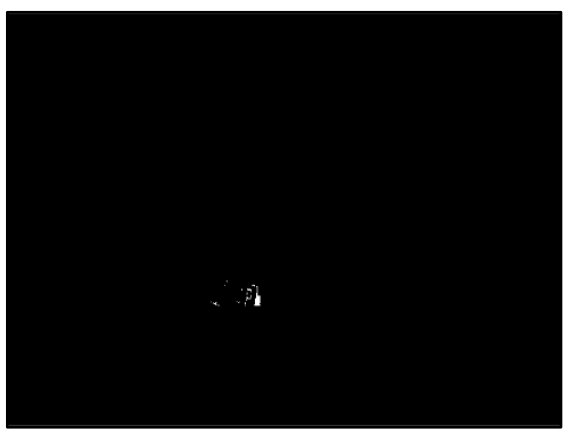

(a)

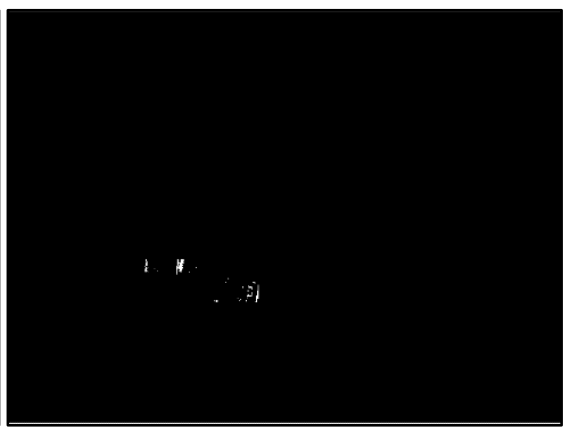

(b)

Figure 8

(a) Extracted content based on the binary mask of the GFBank and (b) extracted content based on the binary mask of the FuzzyGF

Fig. 8 displays the binary content of the images in both examples, extracted with the appropriate binary masks [1], [5], [10].In Fig. 8 (a), where the filter bank with crisp parameters was used, the content has been marginally cropped on the left side of the plate and the content of the plate is not visible in the binary image. In Fig. 8 (b), the entire plate is extracted, but the content of the plate is not visible due to the poor quality of the input image.

Finally, after completion of the morphological filtering and connected component analysis [1], [5], [10] the license plate was extracted and cropped from the original colored image. As demonstrated on the provided example, the improvement of the algorithm based on the FuzzyGF is significant, as the content of the plate is entirely extracted and can easily be recognized. In Fig. 9 (a), the loss of two almost entire characters in this example renders the recognition of the license plate impossible. The reason for such a result is an extremely sharp angle, for which the fuzzified filter provides a superior response. This result proves the potential of the fuzzification of the Gabor filter parameters in the pre-processing step for the detection of the vicinity of the license plate.

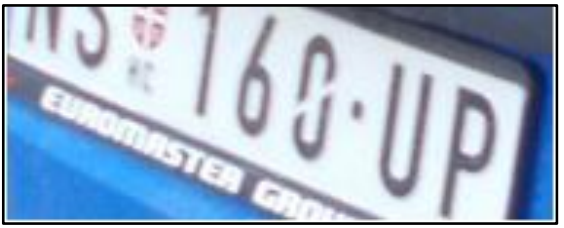

(a)

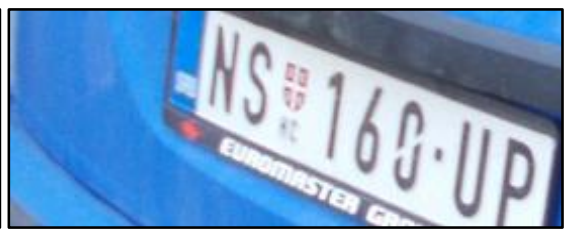

(b)

Figure 9

(a) Plate extracted with a GFBank and (b) plate extracted with a FuzzyGF 


\section{Validation of the Algorithms}

Both algorithms were validated on a dataset of 718 vehicle images captured with a classical modest-quality digital camera [http://appl-dsp.com/wpcontent/uploads/2019/06/test\%20database2.zip] [1]. The images are taken both during a day, and during night, with poor illumination conditions. Also, the assumption is that the slant of the license plates is approximately in interval [-10 10] degrees. The distance between the vehicle and camera is between $2-10 \mathrm{~m}$. The percentage of correctly localized license plates is a commonly used measure for the evaluation of algorithms for license plate detection. Table 3 presents a performance summary of the two compared procedures. The performance improvement, as measured by the percentage of precise detection and extraction of a plate from a complex image has been noted in a fuzzified approach used in FuzzyGF.

Table 3

Summary of the detection results for different pre-processing approaches in FuzzyGF and GFBank

\begin{tabular}{|c|c|c|c|}
\hline Algorithm & Number of Images & Correctly localized & Error (Miss Rate) \\
\hline \hline GFBank & 718 & $94 \%$ & $6 \%$ \\
\hline FuzzyGF & 718 & $97.9 \%$ & $2.1 \%$ \\
\hline
\end{tabular}

The performance of the two presented and compared algorithms is similar to the performance of other license plate detection algorithms [36], [37]. For example, the algorithm proposed by Tan et al. [36], uses the morphological operations for detection and separation of the license plate region similarly to the algorithms compared in this paper. The algorithm pipeline proposed in [36] starts with a contrast enhancement, followed by a difference of Gaussians to improve the edge visibility, and application of a vertical Sobel detector combined with morphological operations to extract the binary mask. As a final step, the geometric properties of the detected areas are extracted and forwarded to the rule-based candidate filtering as a validation step to identify the license plate candidate [36]. The need for a more complex procedure in [36] was imposed by a specific problem of license plate detection in Malaysia. Namely, the plates in this country differ by type, fonts, character ordering and fonts, with additional personalized possibilities. The performance of this algorithm, as evaluated on the proprietary Malaysian database, is $96.9 \%$ of successful detection [36]. It is worth noting that the database images were captured under predefined conditions with respect to the angle and distance to control the experiments [36]. The reported performance of $96,9 \%$ of successful localization is comparable to results presented in this paper.

The development of a universal algorithm providing robust results regardless of the illumination conditions, image quality, capturing settings, and license plate's type would be a challenging image processing task. Imposing some limitations to any of these variables are needed to produce an image processing algorithm with a stable performance. In the low enforcement applications, the controlled 
illumination, capturing distance and angle, and image quality (by using a special REG cameras) ensure robust performance. With the commercial image qualities, as used in this work, the limitations have to be imposed at least on the capturing settings (distance and angle), with similar examples in [1], [2], [5], [11], [18], [25], [28], [36].The fair comparisons of the license plate algorithms developed under the similar assumptions and for the predefined settings would be possible only over the same image database. However, such an exhaustive comparison is beyond a scope of this paper.

The FuzzyGF used in this work has achieved a markedly better performance as compared to GFBank. A plausible explanation is an improved robustness to the image capturing conditions enabled by a fuzzy reasoning. In the applications where some parameters are imprecisely or incorrectly set (such as the input image for detection of license plates, where the precise angle of shooting, brightness, etc. are not known in advance), the use of the fuzzy logic is a good solution, since the fuzzy system successfully uses human reasoning in solving the problem [1], [9], [10], [37]. In the reality, the boundaries between the wavelength and the angle values may be ambiguous and imprecise, and it is difficult to determine whether an input wavelength or angle belongs completely to a certain interval. For that reason, using the concept of the fuzzy set theory [1], [6], the GFBank parameters are replaced with Bell's and triangular fuzzy membership functions within the Gabor filter's parameter calculation [1], [37], [38]. In practice, any membership function can be chosen to describe the similarities between two data sets. As there are no strict boundaries in the fuzzy membership functions and as the membership functions vary continuously and smoothly around chosen values, FuzzyGF performs robustly to slight changes in the wavelength and the angle values.

\section{Conclusions}

This paper evaluates the performance improvement in the algorithm for detection of the license plates in complex images when different filtering procedures in the pre-processing step are applied. The first validated pipeline in a pre-processing block relies on the Gabor filter bank with distinctly crisp parameters, while the other uses the Gabor filter with the fuzzified parameters. The fuzzy logic provides the possibility to advance the problems faced in signal and image processing closer to human reasoning. Both algorithms for the detection of license plates in complex vehicle images, captured with a commercial digital camera, were validated on the same dataset and presented using an example of license plate extraction. In the analysis of the intermediate results, it was evidenced that the GFBank with distinctly crisp parameters would detect and extract the plates with a certain loss, which would later render the full recognition of the characters on the plates impossible. Owing to the method used to capture the images and the modest characteristics of the commercial camera, the initial images included numerous artefacts, reflections, and shadows. The images in the database used in this paper are of commercial, thus lower quality, than the images captured in professional 
law enforcement applications under controlled ambient conditions using a REG camera.

Future projects will focus on a development of an improved license plate detection procedure addressing different limitations that affect both plate segmentation and character recognition. Finally, it should be noted that the procedures described herein can be used to address many other problems in digital image processing and object recognition. Based on the above results, the main conclusion of this study is that owing to fuzzy reasoning, the FuzzyGF has better detected components of interest in complex images and displayed minimal deviation, compared to the GFBank with distinctly crisp parameters. This robustness to the capture setting was achieved using fuzzy logic, which flexibly adjusts the filter parameters to the current problem, which is not possible using classical mathematical reasoning.

\section{Appendix}

The pseudo-code of the algorithm is given in this appendix. The high level view on the development of the license plate extraction software is amended with the relevant references providing additional details and reasoning in the parameter selection.

\begin{tabular}{ll}
\hline Pseudo-code of the algorithm & \\
\hline \hline A=read (INPUT) & $\|$ reading the input color image \\
A1=grayscale(A) & $\|$ converting to grayscale \\
gabor_response=gabor_filter(A1) & $\|$ Gabor filter: GFBank [5, page 3] or \\
FuzzyGF [1, pages 5-7]
\end{tabular}




\section{Acknowledgement}

This work is supported by the European Union, EFOP-3.6.1-16-2016-00003 project and in part supported by the Ministry of Education, Science and Technological Development of the Republic of Serbia, III43002, III47020.

\section{References}

[1] Vladimir Tadic, Miodrag Popovic, Peter Odry, "Fuzzified Gabor filter for license plate detection", Enginnering Applications of Artificial Intelligence, 48 (C), pp: 40-58, 2016, ISSN: 0952-1976

[2] Hung Ngoc Do, Minh-Thanh Vo, Bao Quoc Vuong, Huy Thanh Pham, An Hoang Nguyen, and Huy Quoc Luong, "Automatic License Plate Recognition Using Mobile Device", International Conference on Advanced Technologies for Communications (ATC), 978-1-5090-2710-1/16, 2016, IEEE

[3] Gurwinder Kaur, ReechaSharma,"A Systematic Performance Comparison of Artificial Intelligence Techniques used for ALNPR System", Research Cell: an International Journal of Engineering Sciences, 17(1), pp: 161-167, 2016, ISSN: 2229-6913

[4] Helli, B., Moghaddam, M. E., "A text-independent Persian writer identification based on feature relation graph (FRG)", Jornal of Pattern Recognition, 43(6), pp: 2199-2209, 2010, ISSN: 0031-3203

[5] Vladimir Tadić, Željen Trpovski, Peter Odry, "License Plate Detection using Gabor Filter Banks and Texture Analysis", Proc. $9^{\text {th }}$ IEEE International Symposium on Intelligent Systems and Informatics, Subotica, pp. 381-386, September 2011, ISBN: 978-1-4577-1975-2

[6] Zadeh, L. A., "Fuzzy sets”, Inf. Control, Vol. 8, pp:338-353, 1962

[7] József Menyhárt, Róbert Szabolcsi, "Support Vector Machine and Fuzzy Logic", Acta Polytechnica Hungarica, Vol. 13, No. 5, 2016

[8] László Horváth, Imre J. Rudas, "Information Content Driven Model for Virtual Engineering Space", Acta Polytechnica Hungarica, Vol. 15, No. 2, 2018

[9] Gonzales, R. C., Woods, R. E., "Digital Image Processing”, 3 rd Edition, New Jersey: Pearson Prentice Hall, 2008

[10] Gonzales, R. C., Woods, R. E., Steven L. Eddins, S. L. "Digital Image Processing Using MATLAB", $2^{\text {nd }}$ Edition, Gatesmark, LLC, 2009

[11] Shouyuan Yu, Baopu Li, Qi Zhang, Changchun Liu, MaxQ.-H. Meng, "A novel license plate location method based on wavelet transform and EMD analysis", Pattern Recognition, 48(1), pp: 114-125, 2015, ISSN: 0031-3203 
[12] Juan Climenta, Luiz S. Oliveira, "A new algorithm for number of holes attribute filtering of grey-level images", Pattern Recognition Letters, 53(C), pp. 24-30, 2015, ISSN: 0167-8655

[13] WorawutYimyam, MahasakKetcham, "The Automated Parking Fee Calculation Using License Plate Recognition System", IEEE International Conference on Digital Arts, Media and Technology (ICDAMT) 2017, DOI: 10.1109/ICDAMT.2017.7904985

[14] Khadija Ahmad Jabar, Mohammad FaidzulNasrudin, "Libyan Vehicle Plate Recognition Using Region-Based Features and Probabilistic Neural Network", Journal of Theoretical and Applied Information Technology, 94(1), pp: 104-114, 2016

[15] K. S. Raghunandan, PalaiahnakoteShivakumara, Hamid A. Jalab, Rabha W. Ibrahim, G. Hematha Kumar, Umapada Pal, Tong Lu, "Riesz Fractional Based Model for Enhancing License Plate Detection and Recognition", IEEE Transactions on Circuits and Systems for Video Technology, Volume: PP, Issue: 99, 2018, DOI: 10.1109/TCSVT.2017.2713806

[16] AsadollahShahbahrami, Babak Abad Foomani, AlirezaAkoushideh, "A Style-Free and High Speed Algorithm for License Plate Detection", $10^{\text {th }}$ Iranian Conference on Machine Vision and Image Processing, 2017, DOI: 10.1109/IranianMVIP.2017.8342372

[17] Rafique, M. A., Pedrycz, W., Jeon, M., "Vehicle license plate detection using region-based convolutional neural networks", Soft Computing, Springer-Verlag GmbH Germany, 2017, doi:10.1007/s00500-017-2696-2

[18] G. L. Corneto, F. A. Silva, D. R. Pereira, L. L. Almeida, A. O. Artero, J. P. Papa, V. H. C. de Albuquerque and H. M. Sapia, "A New Method for Automatic Vehicle License Plate Detection", IEEE Latin America Transactions, Vol. 15, No. 1, Jan. 2017, DOI: 10.1109/TLA.2017.7827890

[19] Mahesh Babu K, M V Raghunadh,"Vehicle Number Plate Detection and Recognition using Bounding Box Method", International Conference on Advanced Communication Control and Computing Technologies (ICACCCT), ISBN No.978-1-4673-9545-8. 2016

[20] Jianing Qiu, Naida Zhu, Yi Wei, XiaoQing Yu, "An Optimized License Plate Recognition System for Complex Situations”, IEEE International Conference ICALIP, 2016, 978-1-5090-0654-0/16

[21] Animesh Chandra Roy, Muhammad Kamal Hossen, Debashis Nag, "License Plate Detection and Character Recognition System for Commercial Vehicles based on Morphological Approach and Template Matching”, IEEE International Conference iCEEiCT, 2016, 978-1-50902906-8/16, 2016 IEEE 
[22] WichaiPuarungroj, Narong Boonsirisumpun, "Thai License Plate Recognition Based on Deep Learning", Procedia Computer Science, Vol. 135, pp:214-221, 2018, DOI:10.1016/j.procs.2018.08.168

[23] Faming Shao, Xinqing Wang, Fanjie Meng, Ting Rui, Dong Wang, Jian Tang, "Real-Time Traffic Sign Detection and Recognition Method Based on Simplified Gabor Wavelets and CNNs", Sensors, 18(10). pii: E3192, 2018, doi: 10.3390/s18103192

[24] Youting Zhao, Zhi Yu, Xiying Li, "Evaluation Methodology for License Plate Recognition Systems and Experimental Results", IET Intelligent Transport Systems, 12(5) pp:375-385, 2018, DOI:10.1049/iet-its.2017.0138

[25] Wang Naiguo, Zhu Xiangwei, Zhang Jian, "License Plate Segmentation and Recognition of Chinese Vehicle Based on BPNN", $12^{\text {th }}$ International Conference on Computational Intelligence and Security, 2016, DOI 10.1109/CIS.2016

[26] Yule Yuan, Wenbin Zou, Yong Zhao, Xinan Wang, Xuefeng $\mathrm{Hu}$, and Nikos Komodakis, "A Robust and Efficient Approach to License Plate Detection” IEEE Transactions on Image Processing, pp:1102-1114, 2016, DOI: 10.1109/TIP.2016.2631901

[27] Tejendra Panchal, Hetal Patel, Ami Panchal, "License Plate Detection using Harris Corner and Character Segmentation by Integrated Approach from an Image", Procedia Computer Science, Vol. 79, pp:419-425, 2016, DOI: 10.1016/j.procs.2016.03.054

[28] LeleXie, Tasweer Ahmad, LianwenJin, Yuliang Liu, Sheng Zhang, " A New CNN-Based Method for Multi-Directional Car License Plate Detection", IEEE Transactions on Intelligent Transportation Systems, 19(2), pp:507-517, 2018, DOI: 10.1109/TITS.2017.2784093

[29] Annamaria R. Varkonyi-Koczy, "Fuzzy logic supported corner detection", Journal of Intelligent \& Fuzzy Systems, 19(1), pp. 41-50, 2008

[30] Ernesto Moya-Albor, Hiram Ponce, Jorge Brieva, "An Edge Detection Method using a Fuzzy Ensemble Approach", Acta Polytechnica Hungarica, Vol. 14, No. 3, 2017

[31] Stefan Cirneanu, Loretta Ichim, Dan Popescu, "Improvement of Optic Disc Localization using Gabor Filters", $41^{\text {st }}$ International Conference on Telecommunications and Signal Processing (TSP), Athens, Greece, 2018, DOI: 10.1109/TSP.2018.8441261

[32] S. BabuDevasenapati and K. I. Ramachandran, "Hybrid Fuzzy Model Based Expert System for Misfire Detection in Automobile Engines", International Journal of Artificial Intelligence, Vol. 7, No. A11, pp. 47-62, 2011 
[33] Tamas Haidegger, Levente Kovacs, Radu-Emil Precup, Balazs Benyo, Zoltan Benyo, Stefan Preitl, "Simulation and control for telerobots in space medicine", ActaAstronautica, Vol. 181, No. 1, pp. 390-402, 2012

[34] Marko Jocic, Endre Pap, Anikó Szakál, DjordjeObradovic, Zora Konjovic, "Managing Big Data Using Fuzzy Sets by Directed Graph Node Similarity", Acta Polytechnica Hungarica, Vol. 14, No. 2, pp. 183-200, 2017

[35] S. Vrkalovic, E.-C. Lunca and I.-D. Borlea, "Model-Free Sliding Mode and Fuzzy Controllers for Reverse Osmosis Desalination Plants", International Journal of Artificial Intelligence, Vol. 16, No. 2, pp. 208-222, 2018

[36] Jinn-Li Tan, Syed A. R. Abu-Bakar, Musa M. Mokji, "License plate localization based on edge-geometrical features using morphological approach", Image Processing (ICIP) $201320^{\text {th }}$ IEEE International Conference

[37] Mosabber U. Ahmed, TheerasakChanwimalueang, SudhinThayyil, Danilo P. Mandic, "A Multivariate Multiscale Fuzzy Entropy Algorithm with Application to Uterine EMG Complexity Analysis", Entropy 2017, 19, 2; doi:10.3390/e19010002

[38] Cheng-Hung Lin, Yong-Sin Lin, and Wei-Chen Liu, "An Efficient License Plate Recognition System Using Convolution Neural Networks", Proceedings of IEEE International Conference on Applied System Innovation 2018, IEEE ICASI 2018- Meen, Prior \& Lam (Eds), ISBN 978$1-5386-4342-6$

[39] AyodejiOlalekanSalaua, Thomas KokumoYesufua, Babatunde Sunday Ogundareb, "Vehicle plate number localization using a modified GrabCut algorithm", Journal of King Saud University - Computer and Information Sciences, https://doi.org/10.1016/j.jksuci.2019.01.011 